\title{
DISCOPATIA CERVICAL NO CÃO. TRATAMENTO CIRÚRGICO ATRAVÉS DE FENESTRAÇÃO VENTRAL. ESTUDO RETROSPECTIVO (1986-1997)
}

\author{
CERVICAL DISK DISEASE. SURGICAL TREATMENT THROUGH VENTRAL \\ FENESTRATION. RETROSPECTIVE STUDY (1986-1997)
}

\author{
João Guilherme Padilha Filho ${ }^{1}$ André Luis Selmi $^{2}$
}

RESUMO

Aproximadamente, $15 \%$ das discopatias em cães acometem a região cervical, sendo a dor o principal sinal clínico. Descreve-se a ocorrência de protrusão de disco cervical em 17 cães, agrupados segundo a raça, sexo, peso, idade, assim como a distribuição quanto à duração dos sintomas, acometimento dos discos intervertebrais (DIV), tempo de recuperação e porcentagem de sucesso, em relação à condição neurológica presente antes da cirurgia. A raça Dachshund representou 29,5\% (n=5), cães sem raça definida, Poodle e Cocker Spaniel Inglês, 17,6\% cada (n=9), Pinscher, 11,8\% (n=2) e Dálmata, 5,9\% $(n=1)$. Destes, $58,8 \%$ eram machos $(n=10)$ e $41,2 \%$, fêmeas $(n=7)$, com peso entre 2 e $29 \mathrm{~kg}$, e idade média igual a 5,8 anos. $O$ quadro neurológico desses animais correspondia à dor e ataxia, com exceção de um cão Dálmata, 11 anos de idade, que apresentava tetraparesia. A duração dos sinais variou de 2 a 90 dias. Os DIV mais acometidos foram $C_{2 / 3}(40 \%), C_{3 / 4}(25 \%), C_{4 / 5}(15 \%), C_{5 / 6}$ (10\%) e $C_{67}(10 \%)$, sendo que alguns animais apresentavam lesões múltiplas. O procedimento foi padrão para todos os animais, através da fenestração e curetagem de todos os DIV abordados pelo acesso ventral, ou seja, de $C_{2 / 3}$ até $C_{6 / 7}$, empregandose para isso instrumental usado para remoção de tártaro dentário (curetas Gracey, curetas McCall, extratores de tártaro S.S. White e McCall). O tempo médio de recuperação foi de 9 a 38 dias, sendo que $100 \%$ deles recuperaram totalmente as funções neurológicas. Conclui-se que a fenestração ventral apresenta excelentes resultados no tratamento das discopatias cervicais, desde que bem selecionados os pacientes, inclusive, com respeito aos diagnósticos diferenciais.

Palavras-chave: discopatia cervical, fenestração ventral, cão.

\section{SUMMARY}

Approximately $15 \%$ of canine disk disease affects the cervical region, with pain being the most significant sign. The

\begin{abstract}
authors describe the ocurrence of cervical disk protrusion in 17 dogs, grouped according to the breed, sex, weight, age, duration of symptoms, disk involvement, time elapsed to recuperation after surgery and results. Dachshund represented 29.5\% ( $n=5)$, mixedbreed dogs, Miniature Poodle and English Cocker Spaniel, 17.6\% each (n=9), Miniature Pinscher, $11.8 \%(n=2)$ and Dalmatian, $5.9 \%(n=1)$. Fifty-eight per cent were males. Weight varied from 2 to $29 \mathrm{~kg}$. Mean age at presentation was 5.8 years. All dogs presented neck pain and various degrees of ataxia, exception of one dog that was tetraparetic at time of clinical exam. Duration of clinical signs varied from 2 to 90 days. Intervertebral disks more commonly affected were $C_{2 / 3}(40 \%), C_{3 / 4}(25 \%), C_{4 / 5}(15 \%)$, $C_{5 / 6}(10 \%)$ e $C_{6 / 7}(10 \%) ;$ some animals presented multifocal involvement. Ventral cervical fenestration was performed in disks $C_{2 / 3}$ to $C_{6 \pi}$, using tartar scrapers for curetage. Mean time for recuperation was beetwen 9 and 38 days, with 100\% of success regarding sensory-motor function. It is concluded that ventral fenestration provides excelent results for the treatment of cervical disk disease, considering case selection.
\end{abstract}

Key words: cervical disk disease, ventral fenestration, dog.

\section{INTRODUÇÃO}

A discopatia cervical é responsável por cerca de $14 \%$ a $16 \%$ das doenças que afetam o disco intervertebral (DIV), e é a segunda forma mais comum de doença desta estrutura nos cães (JANSSENS, 1990; TOOMBS, 1992; BRAUND, 1993; GILL et al., 1996). Raças condrodistróficas e cães de pequeno porte são mais comumente afetadas. As raças Dachshund, Poodle miniatura e Beagle representam aproximadamente $80 \%$ dos casos observados mais freqüentemente nos animais entre

\footnotetext{
${ }^{1}$ Professor Assistente, Doutor, Faculdade de Ciências Agrárias e Veterinárias (FCAV), UNESP. Rod. Carlos Tonanni, Km 5, $14870-000$ Jaboticabal-SP. E-mail: padilha@fcav.unesp.br. Autor para correspondência.

${ }^{2}$ Médico Veterinário, Pós-graduando em Medicina Veterinária, Área de concentração em Cirurgia Veterinária, FCAV, UNESP, Jaboticabal.
} 
quatro e oito anos de idade (JANSSENS, 1990; GILL et al., 1996). DALLMAN et al.(1992) verificaram maior ocorrência na faixa etária compreendida entre três e seis anos.

Os sinais clínicos podem variar desde dor cervical a ataxia, hemiparesia, tetraparesia ou plegia (BRAUND, 1993; GILL et al., 1996). Não é rara a presença de dor cervical associada à claudicação em membro torácico. Freqüentemente, a dor não responde a medicamentos, e apresenta-se como condição que faz com que os cães vocalizem ao menor movimento ou ao serem tocados (WHEELER \& SHARP, 1994). A severidade desses sinais dependem de fatores como localização da hérnia, volume e velocidade do material herniado, bem como do tempo de evolução do quadro (GILL et al., 1996).

$\mathrm{O}$ espaço intervertebral mais acometido é $\mathrm{C}_{2 / 3}$, sendo menor a freqüência à medida que se desloca caudalmente. $\mathrm{O}$ acometimento dos três primeiros discos intervertebrais cervicais representa cerca de $90 \%$ da localização da doença (JANSSENS, 1990). Muitas vezes, o material do disco é herniado em direção dorsal, porém, em alguns casos a direção da hérnia poderá ser no sentido lateral ou intraforaminal, produzindo sinais clínicos diferentes daqueles observados comumente. Nestes casos, observa-se claudicação de apoio ipsilateral à hérnia, principalmente nos casos de compressões de raízes de nervos periféricos (BAGLEY et al., 1996).

O diagnóstico normalmente é realizado através de minucioso exame clínico e neurológico. Normalmente, observa-se espasticidade da musculatura cervical, e exacerbação da dor durante a palpação da região em questão. O diagnóstico é confirmado através de exame radiográfico simples em $60 \%$ dos casos, sendo necessária a mielografia nos outros 40\% (JANSSENS, 1990), que deverá ser realizada após exclusão de outras afecções que podem apresentar a mesma sintomatologia como: discoespondilite, meningite, subluxação atlanto-axial, entre outras (TOOMBS, 1992).

Os tratamentos variam desde restrição ao exercício e administração de corticóides a procedimentos cirúrgicos como fenestração ventral, descompressão ventral, hemilaminectomia e facetectomia por acesso lateral, e laminectomia dorsal (FRY et al., 1991; LIPSITZ \& BAILEY, 1992; BAGLEY et al., 1996; GILL et al., 1996). O presente trabalho objetiva avaliar retrospectivamente os resultados obtidos através da fenestração ventral, como tratamento das hérnias de disco cervicais em 17 cães.

\section{MATERIAL E MÉTODOS}

Foram estudados 17 casos de hérnias de disco cervical em cães atendidos junto ao Serviço de Ortopedia e Traumatologia do Hospital Veterinário da Faculdade de Ciências Agrárias e Veterinárias UNESP - Campus de Jaboticabal, no período de Julho de 1986 a Julho de 1997. Cães que sofreram fenestração cervical ventral, mas foram diagnosticados como portadores de espondilomielopatia cervical, ou aqueles cujos dados para preenchimento dos quesitos do estudo estavam incompletos, não foram incluídos.

As informações obtidas a partir das fichas clínicas incluíam: raça, sexo, idade, massa corpórea, sinais clínicos presentes à consulta, duração dos sintomas, localização dos discos intervertebrais afetados, tratamentos prévios, tempo de recuperação e resultados após a fenestração ventral. Os cães foram classificados em 3 grupos: GI - dor, GII - dor e paraparesia, GIII -paraplegia.

O diagnóstico foi realizado através de exame clínico e neurológico, seguido de confirmação radiográfica baseada na redução do espaço intervertebral, material do disco presente no assoalho do canal medular, no forame intervertebral ou redução do espaço correspondente ao forame intervertebral. Nos casos onde o resultado do exame radiográfico simples era inconclusivo, foi realizada mielografia através de punção da cisterna magna e aplicação do contraste iohexol ${ }^{\mathrm{a}}$.

O procedimento padrão para todos os casos foi a fenestração ventral pela linha média de todos os discos intervertebrais cervicais a partir de $\mathrm{C}_{2 / 3}$ até $\mathrm{C}_{6 / 7}$ que consistiu na incisão do anel fibroso com lâmina de bisturi $\mathrm{n}^{\circ} 15$, removendo-se uma porção retangular deste, seguida de meticulosa curetagem do espaço intervertebral com instrumentos para remoção de tártaro dentário (curetas Gracey, curetas McCall, extratores de tártaro S.S. White e McCall). O pós-operatório consistiu na administração de antibiótico (ampicilina ${ }^{\mathrm{b}}$ - 20mg/kg/SC -SID) e analgésico $\left(\right.$ dipirona $^{\mathrm{c}}-20 \mathrm{mg} / \mathrm{kg} / \mathrm{SC}-\mathrm{BID}$ ), além de curativo tópico (rifamicina ${ }^{\mathrm{d}}$ ), durante 7 dias consecutivos.

Os dados relativos à evolução dos animais foram obtidos nas consultas posteriores à cirurgia, aos nove, vinte e um e quarenta dias após a cirurgia. Aqueles animais que, porventura, não compareceram às avaliações pós-cirúrgicas, tiveram seus proprietários contatados por telefone, onde se procurou obter informações sobre a evolução do paciente. Esses contatos foram efetuados 24 meses após a cirurgia, para que se pudesse obter informações relativas à evolução clínica a longo prazo, bem como a ocorrência ou não de episódios recidivantes.

\section{RESULTADOS}

As hérnias de disco cervical foram observadas em 10 cães machos $(58,8 \%)$ e 7 fêmeas $(41,2 \%)$, com idades variando entre dois e treze anos 
( $\mathrm{X}=5,8$ anos) e massa corpórea entre 2 e $29 \mathrm{~kg}$. Destes, $58,82 \%$ tinham entre três e seis anos de idade.

Entre as raças de cães observadas neste estudo, tem-se maior representação da raça Dachshund, seguida pelos animais das raças Poodle miniatura, Cocker Spaniel Inglês, cães mestiços, Pinscher e Dálmata. A maioria dos cães pertenciam a raças condrodistróficas $(76,48 \%)$.

Os sinais clínicos variaram entre dor (GI 7 animais), paraparesia (GII - 7), paraplegia (GIII 3). A duração média dos sinais clínicos, em dias, para cada grupo foi: GI - 33,6 (7-120 dias), GII 24,1 (1-90 dias), GIII - 3,6 (3-4 dias). O sinal clínico mais evidente foi dor cervical, presente em todos animais. Todos os cães apresentavam sensação de dor profunda intacta. Um animal do GII apresentava tetraparesia não-ambulatória ao exame clínico inicial.

Os discos intervertebrais mais acometidos foram, em ordem decrescente, $\mathrm{C}_{2 / 3}-40 \%, \mathrm{C}_{3 / 4}$ $25 \%, \mathrm{C}_{4 / 5}-15 \%, \mathrm{C}_{5 / 6}-10 \%$, e $_{6 / 7}-10 \%$. Lesões múltiplas estavam presentes em três animais. $\mathrm{O}$ tempo de recuperação após fenestração ventral, para cada grupo, foi: GI - 12,2 (9-17), GII - 18,6 (13-32), GIII - 32,3 (23-38) dias. Após a cirurgia, 94,2\% dos animais apresentaram remissão completa dos sinais clínicos, exceção a um cão pertencente ao GIII, que apresentava dor durante a ventroflexão cervical aos 80 dias após a cirurgia. Este animal foi considerado normal após contato telefônico com seu proprietário. Nenhum animal apresentou recidivas dos sinais dentro do período de 24 meses após a cirurgia.

\section{DISCUSSÃO}

Várias técnicas têm sido descritas na literatura como forma de tratamento das hérnias de disco cervicais nos cães (FRY et al., 1991; LIPSITZ \& BAILEY, 1992; BAGLEY et al., 1996; GILL et al., 1996). O objetivo da fenestração ventral é a remoção do núcleo pulposo através de uma janela produzida cirurgicamente na face ventral do anel fibroso do disco intervertebral, de modo que o canal vertebral não seja penetrado. Segundo TOOMBS (1992), a eficiência da fenestração depende da completa remoção do núcleo pulposo, obtida com sucessivas curetagens do espaço intervertebral.

A idade média dos cães, neste estudo, aproxima-se dos dados divulgados por DALLMAN et al. (1992), que descrevem maior prevalência em cães entre três e seis anos de idade. Quando se relacionou a idade com os sinais clínicos, não foi observada qualquer diferença percentual significativa.
A maioria dos cães pertencia a raças condrodistróficas, com maior representação da raça Dachshund, seguida por Poodle miniatura, Cocker Spaniel Inglês, cães sem raça definida, Pinscher e Dálmata, assim como o observado por FRY et al. (1991) e GILL et al. (1996). Não foi observada predileção sexual, o peso não foi considerado fator desencadeante, nem demonstrou estar relacionado com a gravidade dos sintomas.

Em geral, o sinal clínico mais observado e característico é a dor, sendo relativamente rara a presença de disfunções neurológicas. Tal fato é explicado pela relação do diâmetro entre o canal medular e a medula espinhal; na região cervical, a medula ocupa menos espaço no canal medular do que na região torácica e lombar (TOOMBS, 1992). $\mathrm{O}$ espaço intervertebral mais afetado foi $\mathrm{C}_{2 / 3}$, sendo decrescente a representação dos demais discos intervertebrais, à medida que eram localizados caudalmente. Tais achados foram também observados por outros autores (WATERS, 1989; FRY et al., 1991; DALLMAN et al., 1992).

Vários fatores devem ser considerados quando da opção ao melhor tratamento para as hérnias de disco cervical. O tratamento conservativo, baseado em corticóides e repouso, apresenta resultados favoráveis em grande número de animais tratados, porém a taxa de recidivas da sintomatologia tem sido descrita como sendo em torno de 33\%. Geralmente, os animais tratados pelo método conservativo apresentam agravamento dos sinais clínicos nos episódios recidivantes. Tal agravamento deve-se ao fato de que, muitas vezes, o cão recebe droga antiinflamatória e passa a apresentar melhora significativa do quadro clínico, porém a falta de repouso, muitas vezes não realizada corretamente, faz com que não ocorra cicatrização das fibras lamelares do anel fibroso, tendo como consequiência a ruptura e extrusão do material nuclear (TOOMBS, 1992).

FRY et al. (1991) compararam os resultados em 111 casos de hérnia de disco cervical tratadas através de fenestração ou descompressão ventral e concluíram que a recuperação dos animais tratados por descompressão apresentaram evolução pósoperatória mais rápida, sem diferença estatisticamente significativa quanto à porcentagem de recuperação dos animais tratados por ambos os métodos. Além do mais, a incidência de complicações trans e pós-operatórias foi maior no grupo tratado por descompressão ventral.

Segundo FRY et al. (1991) e GILL et al. (1996), a descompressão ventral apresentou como desvantagens: limitação do tamanho da descompressão, especialmente em cães de diminuto porte, e risco de lesão iatrogênica aos seios venosos verte- 
brais, apesar de ser técnica amplamente utilizada nos casos de extrusão de DIV, por permitir remoção cirúrgica do material herniado de dentro do canal vertebral.

GILL et al. (1996) descreveram os resultados obtidos através de laminectomia dorsal no tratamento das hérnias de disco cervical, com resultados satisfatórios a longo prazo, porém, avaliandose os dados obtidos por esses autores, nos 14 dias que sucederam à cirurgia, observa-se que todos animais apresentavam dor cervical, independentes do quadro clínico inicial. Apesar do procedimento descompressivo ser de grande valia no tratamento das extrusões de disco, tal procedimento requer instrumental cirúrgico especializado, é tecnicamente mais difícil, o tempo cirúrgico é prolongado, há necessidade de correta localização da hérnia, e risco de trauma iatrogênico à medula espinhal. Os resultados obtidos no presente estudo, mostram que a fenestração ventral foi excelente alternativa para o tratamento das hérnias de disco cervicais, uma vez que produziu resultados satisfatórios, sem que fosse necessário maior trauma tecidual, como é o caso dos procedimentos descompressivos. A duração dos sinais clínicos foi diretamente relacionada ao tempo de recuperação dos animais.

O aparecimento de tetraparesia nãoambulatória parece ser manifestação bastante incomum da hérnia de disco cervical. Segundo WATERS (1989), a ocorrência de tetraparesia nãoambulatória é observada em $17,6 \%$ dos casos de hérnias cervicais. No presente estudo, apenas um cão da raça Dálmata, macho, 13 anos de idade, apresentou este quadro. Radiograficamente, pôde ser observado presença de material de disco calcificado no assoalho do canal vertebral entre $\mathrm{C}_{4}$ e $\mathrm{C}_{5}$, tendo recuperado completamente as funções neurológicas após a fenestração dos discos compreendidos entre $\mathrm{C}_{2 / 3}$ e $\mathrm{C}_{6 / 7}$. O tempo de recuperação completa dos pacientes, porém, foi mais demorado quando comparado à descompressão ventral, realizada por outros autores (FRY et al.,1991; DALLMAN et al., 1992).

Outros procedimentos como a descompressão lateral ou hemilaminectomia/facetectomia ainda não apresentam resultados a longo termo, a fim de que possam ser comparadas com outras formas de descompressão medular, além de serem indicadas principalmente em casos de hérnias laterais ou foraminais, o que não foi observado pelos autores.

\section{CONCLUSÃO}

A fenestração ventral produz bons resultados com taxa de recidiva nula, apresentando-se como excelente alternativa no tratamento das hérnias do disco intervertebral cervical, desde que bem selecionados os pacientes, principalmente quanto aos diagnósticos diferenciais.

A fenestração ventral é técnica relativamente simples, não requer instrumental específico ou oneroso, sendo portanto passível de ser empregada pela grande maioria dos cirurgiões veterinários.

\section{FONTES DE AQUISIÇÃO}

a - Omnipaque. Sanofi Winthrop Farmacêutica Ltda.

b - Optacilin 500. Laboratórios BYK.

c - Novalgina injetável. Hoechst do Brasil.

d - Rifocina spray. Merrel Lepetit.

\section{REFERÊNCIAS BIBLIOGRÁFICAS}

BAGLEY, R.S., TUCKER, R., HARRINGTON, M. L. Lateral and foraminal disk extrusion in dogs. Compendium Continuing Education Practicing Veterinarian, v. 18, n. 7, p. $795-804,1996$.

BRAUND, K.G. Intervertebral disk disease. In: BOJRAB, M.J. Disease mechanisms in small animal surgery. Philadelphia: Lea \& Febiger, 1993. Cap.129. p. 960-970.

DALlMAN, M.J., PALETTAS, P., BOJRAB, J. Characteristics of dogs admitted for treatment of cervical intervertebral disk disease: 105 cases (1972-1982). Journal American Veterinary Medical Association, v. 200, n. 12, p. 2009-2011, 1992.

FRY, T.R., JOHNSON, A.L., HUNGERFORD, L., et al. Surgical treatment of cervical disc herniations in ambulatory dogs. Ventral decompression vs. fenestration, 111 cases (19801988). Progress Veterinary Neurology, v. 2, n. 3, p. 165173,1991

GILL, P.J., LIPPINCOTT, C.L., ANDERSON, S.M. Dorsal laminectomy in the treatment of cervical intervertebral disk disease in small dogs: a retrospective study of 30 cases. Journal American Animal Hospital Association, v. 32, p. $77-80,1996$

JANSSENS, L.A.A. Canine cervical disc disease: a review. Tijdschrift Diergeneeskunde, v. 115, p. 199-206, 1990.

LIPSITZ, D., BAILEY, C.S. Lateral approach for cervical spinal cord decompression. Progress Veterinary Neurology, v. 3, n. 1, p. 39-44, 1992.

TOOMBS, J.P. Cervical intervertebral disk disease in dogs. Compendium Continuing Education Practicing Veterinarian, v. 14, n. 11, p. 1477-1487, 1992.

WATERS, D.J. Nonambulatory tetraparesis secondary to cervical disk disease in the dog. Journal American Animal Hospital Association, v. 25, p. 647-653, 1989.

WHEELER, S.J., SHARP, N.J.H. Small animal spinal disorders: diagnosis and surgery. London: Mosby-Wolfe. 1994. Cap.7: Cervical disc disease: p. 68-84 